\title{
Research integrity and misconduct: a clarification of the concepts
}

TD Khanyile, PhD

School of Nursing University of the Western Cape

S Duma, M.Cur

Division of Nursing, School of Rehabilitative Health Sciences, UCT

LP Fakude, M.Sc (Nursing)

School of Nursing, University of the Western Cape

N Mbombo, PhD

School of Nursing, University of the Western Cape

F Daniels, MPH

School of Nursing, University of the Western Cape

MS Sabone PhD

Department of Nursing, University of Botswana

\section{Keywords:}

research integrity, misconduct, scientific community, randomized response techiniques and self regulation

\section{Correspondence address:}

Prof T Khanyile

School of Nursing

University of the Western Cape

P/B X 17

Bellville

7530

Tel : +27219593003

Fax: +27219591482

Email:tkhanyile@uwc.ac.za

\section{Abstract: Curationis 29(1): 40-45}

The commercialization of research and the ever changing scientific environment has led scholars to shift the focus from promoting research integrity to regulating misconduct. As a result, most literature explains research integrity in terms of avoidance of misconduct. The purpose of the paper is to stimulate reflection and discussion on research integrity and research misconduct. This article explores the meaning of research integrity and research misconduct, and how research integrity can be promoted to ensure safer research and scholarship. We believe that the discussion can help clarify some hazy areas in the research and publication processes, and appreciate some crucial aspects that they may have seen taken for granted. The purpose of this article is to share with the readers some clarification or analysis of the two concepts namely: research integrity and misconduct. The objectives are: (1) To explore and analyse the concepts of research integrity and research misconduct from the educational or developmental perspective and not the legal perspective as others in literature have done. (2) To stimulate the reflection and discussion on strategies to promote research integrity and thus prevent research misconduct

Literature review and concept analysis was undertaken to clarify the two concepts. We argue that the two concepts can be viewed along a continuum, i.e. where research integrity ends, research misconduct starts. We also argue that it is the responsibility of the research community at large to always ensure that the scientific ethics balance is maintained throughout the research process to ensure research integrity and avoid research misconduct. We also argue that research integrity is interlinked with morality while misconduct is interlinked with immorality. 


\section{Background to the problem}

Researchers in higher education environments are continuously threatened with the "publish or perish" statements. It has been observed that in their pursuit of this, some have resorted to short cuts. As a result, the higher education research authorities find themselves having to monitor and regulate research misconduct at the expense of promoting research integrity. As pressure for research and publication in academia continues to mount, one needs to pause and reflect, or else risk ending up with a product, the ingredients and methods for which one can hardly speak to. One common feature in this research and publication race is collaborative research that has become an attractive enterprise for senior and junior lecturers alike, locally and internationally. We may be exploring some short cuts, but the bottom line is: we all want to do it right and to do the right thing. How do we proceed safely in this race so that our research findings can be trusted, further developed by others and implemented for the improvement of nursing care? Literature is abound with information on what constitutes misconduct. Higher Education Institutions as knowledge production sites have all developed policies and guidelines on Research Ethics. What is interesting when these are evaluated is the emphasis on misconduct and not integrity. Therefore the authors through this article will attempt to highlight and clarify the significance use and application of the two concepts.

\section{Strategies for Concept Clarification}

A review of literature from 1985 to 2005 was compiled with a focus on research integrity and misconduct and scientific ethics. The primary purpose was to examine the meaning of the two concepts as reflected in literature. The aim of the concept analysis was to clarify the concept of research integrity and misconduct. Among other things, concept analysis improves practice by offering users a clearer understanding of what certain terms mean (Chinn \& Kramer 1991:281). Walker \& Avant (1995: 67) reported three products that can emerge from analyzing a concept. These are antecedents, defining characteristics and operational definitions. This method is criticized as being reductionist (Rogers, 1989: 200). The constructivists argue that a holistic approach to concept development has three phases, which are: significance, use and application. According to Rogers (1989), the significance of an existing concept plays an important role in the continuing development. He focuses on the meaning, the use, and the significance of the concept. For the purpose of this article, the authors adopted Roger's approach to clarify the two interdependent concepts namely research integrity and misconduct. The authors view these two concepts as opposite ends of a continuum of research ethics. Where research integrity ends, misconduct begins and the basis of the two is scientific ethics.

Literature review revealed the following: the meaning and examples of research integrity, and research misconduct, few studies done on research ethics and different models for promotion of research integrity. These form the basis for our argument and our recommendations to the research community.

\section{Meaning of research integrity}

Research integrity is simply, justice and honesty in proposing, conducting, and reporting research or doing it right and telling the truth about what you did. In other words it means that one conducts one's research as carefully as one can and present the results as honestly as one can. Integrity in research embraces the aspirational standard of scientific conduct rather than simply the avoidance of questionable practices (Swazey 1993: 202; King and Anderson, 1999:34; Steneck, 2003:401; Iverson and Siang, 2003:64; Whitbeck, 2004:85).

We argue that this definition of research integrity puts more responsibility on the researcher or the scientist and not the research communities such as the higher education institutions. It also focuses on the conduct of research and not the research environment in which the researcher might find himself or herself. Bolton (2002: 67), defines research integrity as:

..." the process of doing and reporting science in accordance with accepted practices in their field. This includes adherence to the principles and practices of scientific standards, education and mentoring, unbiased peer and expert review and communication of results to the scientific community". This definition of research integrity seems to be aligned with our belief that not only the researcher, but also the environment and the whole research community is responsible for the promotion of research integrity.

The most basic tenets of research integrity are: trustworthiness and credibility of the findings. (Swazey, 1993: 2002; King, and Anderson 1999: 37). Research integrity should be an ethic that is transmitted down the generational tree, nourished and sustained through individual self-reflection and a continuous bi-directional vertical and horizontal communication among members of the scientific community (Bolton, 2002:78). It should be validated through a continuous dialogue with society because the society is a major stakeholder in addressing the issues of research integrity. The aim of research ethics is to protect members of society as well as to protect the integrity of science itself.

According to the Natal Academic Press (1999), promotion of integrity in the research environment is about institutional culture and behavior, as well as the professional performance of individuals. We argue and propose that research integrity be viewed positively, from an educational or developmental perspective rather than from a regulatory perspective. We believe that only positive messages will assist researchers in their endeavour to maintain research integrity all the time.

Bolton, (2002: 101) proposes the following aspects of integrity during the conduct of research. These are grouped together as follows:

- Childhood socialization. As young children we acquire a moral sense of right and wrong. It is expected that as professionals we will accept the professional standards about data falsification, fabrication and plagiarism

- Scientific socialization. Student's are socialized through education and training of acceptable standards of conducting research.

- Collegial and professional norms. This involves mentoring of junior researchers and postgraduate students. 
Workplace norms, values and incentives. The workplace "culture" will influence the attitude and the ways in which the rules and standards about research integrity are managed (Bolton, 2002:19)

We support Bolton on these aspects. We further argue that the scientific community is another aspect that can play a crucial role in research integrity promotion. We believe that the concept 'scientific community' needs further exploration as well, since scientific ethics forms the basis of research integrity or misconduct.

\section{Who is the research community?}

According to Frankel (1993:234), all those that have the ability to promote scientific integrity and have a role to play in oversight of research and in controlling scientific misconduct from the research community. They include the following:

\section{- Institutions who employ} researchers as academics and scientist who therefore have a responsibility for ensuring that these researchers comply with policies and procedures on the conduct of research.

- $\quad$ Editors and publishers of scientific articles who have an interest in being the first to publish ground- breaking science and also have an interest in enhancing the reputation of the publishing institution. Editors and publishers are charged with a responsibility of critiquing and disseminating the research, therefore they are in a unique position to help cultivate a scientific culture that promotes research integrity through the instructions they provide to authors. Editors also have a responsibility to enhance the research cycle by educating their readers about research integrity. Such efforts not only assist the author but may prove to be effective in promoting the journal's integrity (Schultz, 2000 ). Scholarly journals comprise a vital part of the research process and serve multiple functions for the research community. Journals communicate knowledge, symbolize the currency by which researchers build careers and legitimize research, therefore the journal is valued as reliable information medium by which the research community depend for advancement in science and for public good (Schultz, 2000; Steneck, 2003; Farthing, 1998).

- Scientist or researcher themselves who also serve as reviewers, colleagues, consumers of other's research results and members of professional associations.

- $\quad$ Funding agencies that commission research and thus have a responsibility to ensure that funds are effectively used.

\section{Studies done on research ethics}

Freda and Kearney (2005) conducted a survey of nursing editors via e-mail. From the content analysis of survey questions about ethics, eight categories of ethical issues emerged: problems with society/ association/publisher; decisions about inflammatory submissions; informed consent; conflicts of interest; advertising pressures; duplicate publications and/or plagiarism; difficult interactions with authors; and authorship. They recommended that professional discussions about ethics in publications should be the subject of ongoing research and scientific inquiry. Integrity should also be interpreted to include the ethical treatment of all collaborators, assistants, students, and employees associated with the research effort.

Bailey, Hasselback \& Kacher (2005:28) conducted a survey to determine the reason scientists engaged in misconduct. According to the results fewer scientists $0.2 \%$ plagiarize or falsify. However, $4.7 \%$ scientists admitted to publishing the same data in two or more publication to beef up their resources. A total of $13.5 \%$ admitted to employing research designs they knew before hand would not yield accurate results.

\section{Meaning of misconduct}

Research misconduct is concerned with fabricating, falsifying, plagiarizing, or any other practices that seriously deviate from standard acceptable within the scholarly scientific community in proposing, conducting and reporting research (University of California, Los Angeles Policy 993, 1998).

The White House Office of Science and Technology (OSTP), defined misconduct as:

"fabrication, falsification or plagiarism in proposing performing or reviewing research or in reporting research results". The policy also states that findings of misconduct require that there be significant departure from acceptable practice. It also states that allegations be proven by a preponderance of evidence. It also makes reporting of misconduct to be the primary responsibility of the institution where research is conducted (Guenin 1999: 340).

Most definitions on misconduct highlight the following errors:

- Fabrication: which is defined as making up results and recording and reporting them with a deliberate intent to deceive thus disregarding the accepted scientific practice. It also involves changing (manipulating) data or experiments or the conditions to make results "fit" the hypothesis (Guenin: 1999:342). Other temptations in research may be failure to report research findings that contradict those being reported and failure to report personal interest that the researcher may have in the outcome of the research.

- Falsification: Manipulating research materials, equipment, or process or changing or omitting data or results such that the research is not accurately represented in the research record (Guenin: 1999:342).

- Plagiarism: Appropriation of another person's ideas, processes, results or words without giving appropriate credit, including those obtained from confidential review of others' research proposals and manuscripts. Researchers may also steal data from students, colleagues, and protégés. Plagiarism includes stealing own work or failure to reference own prior work (King and

Anderson, 1999; Swazey, 1993). 
When we take a closer look at the above definitions of research misconduct, it becomes clear that for any research activity to fall under misconduct, the following should occur:

(a) Significant deviation from accepted standards of conducting research The activity must be conducted intentionally for instance with an intention to deceive.

(c) The scientific community has an important role to play.

In our opinions and those of King and Anderson (1999); Swazey (1993); UCLA Policy (1998), research misconduct does not include honest error or difference of opinion or different interpretation of data.

There are other practices besides the violation of the standards accepted by the scientific community that are also classified as research misconduct. These are labelled as "other forms of professional misconduct related to research." in order to underscore the seriousness of research misconduct from research integrity separated such practices from research misconduct (USA Ryan Commission, undated publication). Ryan Commission argues that the definition of research misconduct must reflect research misconduct as a serious violation of the fundamental principle that scientists be truthful and fair in conducting research and disseminating its findings.

Other forms of misconduct related to research cover two areas, namely: (a) obstruction of investigations of research misconduct such as destroying the evidence or retaliating against the whistleblowers, and (b) failure to abide by research regulations or the requirements of the review boards such as handling of bio-hazardous materials and protection of subjects in research.

Fraud means serious misconduct with intent to deceive, but we all know that it is very difficult to detect it since communicating the results of research is always guided by the rules of the scientific journals where these are published. For instance articles that finally reach the public are those that described investigations logically in accordance with the publishers requirements and not in accordance with what actually happened (Goodstein, 2002).

We argue and propose that, researchers must strive to report what they have done and what they have found irrespective of whether or not things did not progress smoothly and whether or not hypotheses tested are supported. Researchers need to be critical of their own work and honestly declare limitations of their studies. Due credit must be given to those whose work is used to support the study. Persons who have significantly contributed to the study must be acknowledged, and this may include coresearchers, data collectors, and funding agencies. Self-plagiarism or failure to acknowledge own prior work also constitutes research misconduct.

\section{The role of publishers in promoting research integrity}

Since science is by nature self- correcting the challenge lies with the editors of the journals that communicate these scientific findings to the public. Journal editors should develop guidelines and policies to promote responsible authorship. These should include procedures for responding to allegations or indications of misconduct in published research. Many journals have specific guidelines and clear policies on how to deal with cases of misconduct but without communicating these to authors and readers, the integrity of the journal is compromised. For instance, a number of South African based journals that are frequently used by nurse academics were reviewed to determine the extent to which their sections on Instructions for Authors dealt with themes to promote research integrity. It was interesting to find out that most of the information covered dealt with manuscript preparation. None of the three journals perused addressed issues pertaining to authorship, peer review on research misconduct. This is important information especially because junior academics rely on this information to guide the manner in which to report their research findings. Scholarly journals comprise a vital part of the research process and serve multiple functions for the research community. Journals communicate knowledge, symbolize the currency by which researchers build careers and legitimize research, therefore the journal is valued as reliable information medium on which the research community depend for advancement in science and for public good (Schultz, 1999).

American Association for the advancement of Science in Washington conducted a survey on 20 scientific research societies that conduct research
(Iverson, Frankel, and Siang, 2003). They were assessing whether the societies were promoting research integrity among their members. They argued that scientific research societies can play an important role in promoting ethical research practices among their members. The study findings suggest that although many of the societies are working to promote research integrity through ethical codes and activities, they lack rigorous assessment methods to determine the effectiveness of their effort. Iverson, Frankel, and Siang (2003:145) endorse reporting of misconduct by editors and reviewers of journals as "dishonest work damages all of science". They argue it is in the interest of individual scientist as well as the community of science to have procedures on how to report misconduct, for example: reporting the individual to home institution.

Goodstein (2002:41) asserts that there are three main motivators for scientists to commit misconduct. The first one being career pressure (scarce resources: research posts and the amount of research funds available). The second being perceived knowledge of what the result would be if they went to all the trouble of doing the work properly. The third one being the difficulty in proving " misconduct' due to the irreproducibility of data as evidence to support allegations of misconduct.

\section{The role of Institutions where research is produced.}

One additional area that researchers and research institutions have not addressed is doing harm through research, the area concerned with research on sensitive topics. Often research does harm to the research participants and the communities that they represent. Research reports may tarnish the image of researchers and the institutions that they represent. Researchers must strive to maximize benefits and minimize risks to the participants, and where there are competing demands between the advancement of science and the protection of human dignity, the latter must take priority over the former. Any research that violates human dignity may therefore constitute research misconduct. RCR-IMR (undated) adds a caveat here that researchers must not be penalized for unintended or unforeseeable research outcomes that may represent an inherent error of the 
scientific process.

Burd (2005:27) argue that industry officials use the state laws on research misconduct and charge researchers. According to Burd, universities should support the researchers. However these universities find themselves having to maintain the relationship between industry and the institution. This is especially true if there, are financial implications to this relationship. Burd (2005:29) raises some important questions about the role of the universities in protecting their employees in cases of allegations of misconduct. The university should protect both the researcher and the whistle-blower.

The authors also analyzed a number of policies on research misconduct from six universities in South Africa and six internationally. The authors also compared polices from Medical Research Councils in South Africa and abroad. The following questions guided the analysis: (a) Were the two concepts adequately defined in the policy? (b) What is the policy's applicability to the scientific community? It was interesting to see that from all the policies analyzed only $8 \%$ adequately defined the two concepts and also described the roles and responsibilities of the different role players namely the scientists, the institution, the supervisors in case of students and the funding agencies. The rest $(92 \%)$ focused only on misconduct without any definition or explanation of what the role and responsibilities of the different role players were in a case of misconduct.

We propose that allegations of misconduct must be adequately investigated, and efforts must be made to protect both whistle blowers and the respondents (those who are charged with wrong-doing). Alleged transgressors must be treated as innocent until proven otherwise.

Jeffers (2005: 63) proposes the use of the Internal Control Model (ICM) as an innovative framework to guide conceptualization and design of research environment, which promotes integrity in research. The ICM is widely used in business and finance for risk management and promotion of financial integrity within organizations. Information systems have also adopted it to guide the design of network security and minimize risks to information systems in organizations. The ICM provides five interrelated processes as follows: internal control environment, risk assessment, internal control activities, monitoring, and information communication.

\section{Recommendations}

\section{Self - Regulation}

Self-regulation plays an important role in identifying and controlling errors and misconduct. Professions including nursing have traditionally been granted relative autonomy to oversee and correct the behavior of their members. An important feature of self-regulation is the ability of other members to adequately judge the credibility of research findings and influence the acceptance of those findings. According to Bolton (2002), there are three mechanisms that provide the basis for self-regulation, namely peer review, refereed publication and replication of research projects to see if similar outcomes can be achieved. Swan (1993) also recommends replication studies as critical in the whole process of scientific discovery and in reducing fraud.

\section{Mentoring of postgraduate students and junior academics}

We need to create environments in which questions about responsible conduct of research are openly discussed and freely debated. Gunsalus (1997) argues against the belief that student can become professional researcher through osmosis or only through taking single modules on research ethics. Postgraduate students and junior academics need to be mentored to ensure that high professional standard are adhered to.

\section{Analytic research on research integrity}

Most studies on research integrity and misconduct are empirical. Bailey (2001) proposed the Randomized Response Technique (RRT) as the most efficient tool in conducting research on sensitive questions like research misconduct since cases of misconduct are difficult to detect by direct observation. In the RRT the subject is given two sets of questions, one sensitive or incriminating and the other non- sensitive. The RRT uses a random number generated by the subject for example a coin toss or serial number on a piece of currency to determine which question the subject should respond to. In this way only the subject knows which question they answered, but the researcher can derive the estimates concerning the sensitive question (Bailey, et al 2001: 30).

\section{Promote integrity and punish misconduct}

We view the two concepts similar to the concepts: well-being and illness. Wellbeing and illness being determinants of health, whereas integrity and misconduct are determinants of scientific ethics. The scientific community should strive to balance the promotion of integrity while preventing and punishing misconduct. Integrity and misconduct should be viewed as opposite ends of a continuum of scientific ethics.

\section{Research Integrity $\underset{\leftarrow}{\longrightarrow}$ Misconduct Scientific ethics}

\section{Conclusion}

Through this article the authors have made an attempt to highlight the importance of the whole scientific community in addressing the issues of research integrity and misconduct. Researchers as knowledge developers owe it to the societies who are the consumers of this knowledge to have a stewardship responsibility to conform to the conventions of their disciplines. The authors in making these recommendations have only focused on those that are not common. Common recommendation like peer review, education and regulation have not been repeated.

This is the first stage of proposed research on research integrity and misconduct.

\section{References}

AMERICAN ASSOCIATION FOR ADVANCEMENT OF SCIENCE:

Washington White House Office of Science and Technology Policy (OSTP)

AMERICANASSOCIATIONFORTHE ADVANCEMENT OF SCIENCE(AAAS) and the U.S. Office of Research Integrity (ORI). The Role of Activities of Scientific Societies in Promoting Research Integrity. Report of a Conference, April 10, 2000, Washington, D.C. Available URL:

http://www.aaas.org/ssp/dspp/sfr 1/ projects/integrity,htm (as of September 30,2000 ).

BAILEY, CD; HASSELBACK, JR \& KARCHER, JN 2005: Research Misconduct in Accounting Literature: A 
Survey of the Most Prolific Researchers' Actions and Beliefs. ABACUS. 37, (1) 2001

BOSTON COLLEGE, 2004:Principles on research integrity and misconduct. Boston College http://www.bc.edu/ research/rcip/princ

BOLTON, PA 2002: Scientific Ethics.

BURNS, N \& GROVE, SK 1993: The Practice of nursing research: conduct, critique and utilization ended Philadelphia W.B. Saunders.

BURD, S 2005: Scientists See Big Business on the Offensive: The Chronicle of Higher Education, Past chronicle Issues.

http:// chronicle.com/data/articles.dir/art41.dir/issue-16.dir/16a02601.htm

CHINN, PL \& KRAMER, MK 1991: Theory and Nursing: A systematic approach $3^{\text {rd }}$. Mosby Year Book. St Louis

CLARK, E \& MC CANN, TV 2005: Researching Students: An Ethical Dilemma. Nurse researcher. 12 (3), 42-52.

FARTHING, J 1998: An editor's response to fraudsters. British Medical Journal. 316(17): 26-33; see www.bmj.com

FAWCETT, J 1989: Analysis and Evaluation of conceptual Modules of Nursing ended. F.A Davis Co. Philadelphia

\section{FIORIDA INTERNATIONAI UNIVER-}

SITY 1995: Policy on Misconduct in Research. Academic Affairs Policies and Procedures Manual.

http://www.vsu.edu/ugs/regulations manual

FRANKEL, MS 2003: Developing a Knowledge Base on Integrity in Research and Scholarship. Phi Kappa Phi Forum. 83(2): $46-49$

FREDA, MC \& KEARNEY MH 2005: Ethical issues faced by nursing editors. Western iournal of nursing research. [West J Nurs Res] Jun; 27 (4): 487-499.

FROSMAN, B 1999: An Ethical Analysis of the Phenomenon of Misconduct in Research. Acta Oncologica. 38(1): 107 110

GOODSTEIN, DL 2002: Scientific
Misconduct:

http://www.aaup.org/publications/ Academe/2002/02JF/02jfgoo.htm.

GUENIN, LM 1999: Expressing a consensus on candour. Nature. Vol 402. www.nature.com.

INGHAM, JC \& HORNER, J 2004: Ethics and Research. The ASHA Leader. March 16

IVERSON,M; FRANKEL, M \& SIANG, $S$ 2003: Scientific Societies and Research Integrity: What are they doing and How well are They doing it? Science and Engineering Ethics. 9, (2): 41-158.

JEFFERS, B 2005: Research Environments That Promote Integrity. Nursing Research, 54(1): 63-70.

KING, AD \& ANDERSON 1999: The meaning of research integrity. Journal of the Medical and Dental Association 157(4): 254-272.

MELEIS, AI 1985: Theoretical Nursing: Development and Progress, J.B Lippincott. Philadelphia.

NATAL ACADEMIC PRESS 1999: Integrity in scientific research.

\section{OFFICE OF RESEARCH INTEGRITY}

1989: Policy on Research Misconduct: United State Department of Health and Human Services.

ROGERS, BL 1989: concept analysis and the development of nursing knowledge. The evolutionary cycle. Journal of Advanced Nursing. 14: 330 335.

SIMON FRASER UNIVERSITY, 1995: Policy \& Procedures. Policy No. 60.01

SCHULTZ, MD 2000: Promoting integrity through instructions to authors. Office of_Research Integrity. U.S Department of Health and Human Services.

SHERMAN J 2005: Professional Misconduct, Plagiarism, Ethics, Educational materials. New England Review. 26(3): 82-89.

SOUTH AFRICAN SOCIETY FOR NURSING RESEARCH 1996: Ethical standards for nurse researchers. Curationis. 19(1): 74-74.
STENECK, N 2000: Assessing research integrity. Report presented at the ORI conference on research integrity. U.S Department of Health \& Human Services

STENECK, NH 2003: The professional Societies in promoting Integrity in Research. American Journal of Health Behavior. 27 (3). S239-S247.

SWAZEY, J \& ANDERSON, MS 1993: Mentors, advisors and role models in graduate and professional education. Association of academic health centres. Washington D.C.

\section{UNITED STATES PUBLIC HEALTH} SERVICES (PHS): Policy on instructions in the Responsible Conduct of Research (RCR) from the office of Research Integrity 2000.

UNIVERSITY OF COLORADO, 1998: Misconduct in research and authorship. University of Colorado System. Administrative Policy Statement.

WALKER, LO \& AVANT, KO 1995: Strategies for theory construction in nursing $3^{\text {rd }}$ co. California: Appellon \& Laugh

WATSON, SJ 1991: An analysis of the concept of experience. Journal of AduNews 16: 1117-1121.

WEISS, R 2005: Scientists admit to misconduct.

sciencepolicy.colorado.edu/ prometheus/ archives/ science_policy_general/

WHITBECK, C 2004: Trust and the Future of Research. Physics Today. November: 48-53; see: http:// encyclopedie-en.snyke.com/articles/ scholarly_method.html. 